\title{
English Version of CWPT (Color Words Pick-out Test)
}

\author{
Takaki Shimura ${ }^{1 *}$, Eriko Okuyama ${ }^{2}$ and Keiko Togami Evans ${ }^{3}$ \\ ${ }^{1} B M E$ Research Lab. Sosei Ltd., Japan \\ ${ }^{2}$ Hamamatsu Human Research LabLtd., Japan \\ ${ }^{3}$ Lawyer, Australia
}

*Corresponding author: Takaki Shimura, BME Research Lab. Sosei Ltd., Japan.

Received Date: August 06, 2020

Published Date: August 24, 2020

Keywords: CWPT, English Version, neuropsychological test, PCSD, MCI, slight disorder

\section{Background}

CWPT [1-4], which is able to detect a slight disorder of cognitive function in PCSD(Preclinical Stage of Dementia), is attracting attention as a neuropsychological test that allows screening at the stage where we must rely on expensive testing equipment such asPET and MRI. CWPT is a test for identifying colors and remembering episodes simultaneously while reading a story containing Color Words (Appendix 1). Original Japanese Version of CWPT is named CKPT(Color Kanji-character Pick-out Test). Using it, we had examined evidencein Japan, which were presented at two international conferences $[5,6]$ and published in an international journal [7]. Through these presentations and publication, we are asking for collaborative researchers word-wide because CWPT can be easily translated and used in various languages.

\section{Announcement of English Version of CWPT}

The English version of CWPT that we developed not only translates the Japanese version, but also includes two enhancements. The firstenhancement is to double the length of the story. As a result, the tester can be free from the following hassles: the test time for those under 60 is halved, and the test results are doubled.So, you can compare from young people to elderly people within the same criteria. The second enhancement is the improvement of the PowerPoint used by the explainer when the subjects receive the CWPT. Conventionally, the explainers have been given a certain amount of education to ensure the uniformity of the explanation, but there was a tendency forthe test results to vary, due to individual differences amongst the explainers. To solve this problem, we have developed a new tool that incorporates the narrator image and explanation into the PowerPoint used for explanation. As a result, I hope that the explainer's variations will be eliminated.

The tools for the English version of CWPT developed are listed below:

1. English Story and Questions booklet

2. English version PowerPoint with narrator

3. Booklet of tester's manual

4. CWPT Implementation Manual Booklet,

Where 1 is distributed to subjects and $2-4$ is issued to collaborator.

\section{Joint Research Procedure}

Researchers wishing to collaborate should informtheir desired purpose of CWPT application. After that, we will enter into a 
nondisclosure agreement and discuss the purpose and conformity of the CWPT. It is important to note here that the diagnostic criteria obtained in Japan needs to be reconstructed in the country of the collaborator, and the evidence obtained in Japan also needs to be retaken in that country. Next, we will enter intoa Collaboration Agreement and disclose the contents. We will cooperate in the acquisition of funds necessary for research.

\section{Story}

Last Sunday, Renate went swimming with her red bag. She went up the hill and could see the long grey sandy beach below. There were red, pink, and yellow beach umbrellas like flowers.

\begin{tabular}{|lc|}
\hline & Questions \\
1.What color is her bag? & (red, yellow, pink, forget) \\
2. What did she go out for? & (shopping, swimming, skiing, forget)
\end{tabular}

Appendix 1: Sample of Story and Questions of English Version of CWPT. First, the subject reads the story. When the subject encounters colored words while reading, she/he will mark $\bigcirc$ if the printed color and the meaning of the color match, and mark X if they do not match. At the same time, the subject will remember the episode of the story. Next, the subject will respond to Questions without looking at the story. The functional evaluation is obtained by the functions of both parties.

\section{Conclusion}

This study is being further refined and we hope to work with researchers around the world to achieve the completion of these screening tests for preclinical stage of dementia.

\section{Acknowledgment}

We would like to express our gratitude for the fact that the development of the CWPT English version was partially funded by Hamamatsu Agency for Innovation.

\section{Conflict of Interest}

No conflict of interest.

\section{References}

1. Takaki Shimura et al. (2003) Japan Patent No.4887720.

2. Takaki Shimura et al. Germany Patent No.1641813.
3. Takaki Shimura et al. UK Patent No.1649813.

4. Takaki Shimura et al. Korean Patent No.0735674.

5. Takaki Shimura, Eriko Okuyama, Hironori Osugi (2018) A Neuropsychological test (CKPT: Color Word Pick-out Test) to be able to detect slight disorder of prefrontal lobe; classify the level of the preclinical stage of dementia, the 24th International Conference on Neuroscience and Neurochemistry, July 22-24,2018,Birmingham, UK.

6. Takaki Shimura, Eriko Okuyama, Hironori Osugi (2019) A Neuropsychological test for Mild Cognitive Impairment and Preclinical Stage of Dementia, Global Conference on Oral Health \& Mental Disorders, July 01-02 2019,Rome, Italy.

7. Takaki Shimura, Eriko Okuyama and Hironori Osugi (2019) CWPT (Color Words Pick-out Test) Available for Classifying the Slight Disorder on the Preclinical Stage of Dementia, HSOA Journal of Alzheimer's and Neurodegenerative Diseases 5(2): 100028. 